\title{
Design and Implementation of the intelligent management system of large scale sports events
}

\author{
Qingqing Wang \\ Zhengzhou Institute of Technology, Zhengzhou 450044, China \\ wagnqq@163.com
}

Keywords: Competition organization, Information System, Management, Large scale sports event, Public sports

\begin{abstract}
Along with economical and cultural development and strengthen of internationalization tendencies day by day, the par agenesis and the prosperity in many kinds of cultural urges. This purpose of this paper is to identify factors affecting sport event, to use project management knowledge to identify operational tasks and to build up operational model, finally, to analyze China's operation management situation and build up Chinese operation model in sport events. Some research methods have been used, such as literature collecting, investigating methods, questionnaire, and statistical and logical methods.
\end{abstract}

\section{Introduction}

With the fast development of sports industry and the successful bid for 2008 Beijing Olympics, more and more national and international sports events have chosen Beijing and Shanghai as their host places. There is little systematic study and investigation on how to plan and manage these sports events more scientifically, rationally and efficiently. By means of literature study, expert interview and experiencing some large-scale sports events such as the 48th WT.T.C, the 7th National University Sports Games, the 13th, 15th World University Table Tennis Championships, and 2005-2006 LT.T.F. Pro-tours etc, this paper researches and analyses the structure of the events; competition organizing; matters needing attention during competition preparation and planning; communication and coordination between different departments; how to draw and apply the flow charts and PERT diagram in planning and managing competition events. With economic globalizations and scientific technique's development, sport event has shown lots of characters under the influence of marketing and humanization. Sport event has the potential power to impact the society and culture, nature and environment, politic, tourism and economy. Many countries for their effect have chased sport events. Under the background of large scale sports event and the strong desire learning more about sport event, our dissertation is absolutely helpful for this situation [1-2].

At the same time, the view about health care keeping in good health integrates the modern health life tidal current. All these urge sports event's development. In recent years, sports event's quantity conducted by our country increases day after day and the scale and the level are also getting bigger and bigger. The sports event is special event with the clear goal [3]. It needs the person to participate and organize. In order to make sports event's benefit, the administrative action must occur inevitably. Management is the key element in the sports event operation [4]. Treating the sports event as the project, then utilizing project management's knowledge system to analysis and manage sports event, our country sports event's management level will be raised, especially in our country large-scale sports event's successfully holding and the commercial sports event's development. Sports competition's developments not only take competition and entertainment as a goal, but take gaining the economic efficiency as the ultimate development goal. But to gaining the economic efficiency, the reasonable operation and management must be implemented to the sports event. The sport event is operated using project management's mentality. It can strengthen sports event's marketability operation. Simultaneously can also study and transplant the overseas sports event's advanced experience to urge the commercial sports event operating successfully [5]. 
Based on project management's mentality, the research on the large-scale sports event's planning and the organization, the implementation and the control is conducted. Obtaining from the large-scale sport event's start, the sport event's feasibility can be analyzed and the organizations and agencies set up. In implementation stage, the main topic is large-scale sports event's competition management, information management and logistics management. In the control stage, the analysis is mainly carried on to the cost and the risk of large-scale sports event. Carrying project management on entire process of the large-scale sports event, the difficult problem can be solved because the large-scale sports event is significant -responsibility, complex- relation, time- press, resource- limited. The project management is a good method to the large-scale sports event with one-timed disposable duty characteristic.

The Organization and management of Large-scale Sport Events is of Large-scale Sport Events organization and management in a variety of activities and effectively do the work of the organization of Large-scale Sport Events, categorized, with the authorized personnel and the division. The long years of practice organization and management of large-scale sporting events is an extremely complex social system project. Therefore, based on the system of scientific thought and modern systems engineering methodology, to rely on modern science and technology to carry out the organization and management of Large-scale Sport Events is an inevitable choice. Large-scale Sport Events organization and management information system is an integrated use of modern information networks and modern digital technology, by streamlining the internal and external management of its Large-scale Sport Events organization and services, optimization, integration and restructuring to achieve the efficient operation of information systems tools. By using this system to break the constraints of time, space and departmental separation, integration of efficient, high-quality, honest communication of information services for the successful hosting of Large-scale Sport Events.

\section{The Basic Understanding and Statue of Competition Organization of Large Scale Sports Event}

Under the international sports event globalization development background, with the unceasing development, economy and society of our country, in our country's whole sports developmental strategy, especially in gravity change of the sports developmental strategy center in recent years, sports industry, becomes the new sports developmental strategy the hot spot which takes the sports event as the backing, looks the society and the economic development as the main purpose, and drawing expends and develops the new economic growth as the essential target .That large-scale sports event which has the public welfare attribute, becomes the best choice for all levels of the government to promote the urban development and to improve the urban image. This has directly caused the vigorous growth of sports event practices in our country. However, compared with vigorous development of sports event practices, the management level has obvious lag. Especially the large-scale sports event's management is the weakness and the difficulty. We take "Organizing pattern innovation and the optimizing operational practice of large-scale sports event of our country" as the main study object, study and refines the optimizing elementary management theory of large-scale sports event in our country, with the help of sociological social environment theory, western economic public choice theory, process management of management principle theory and so on. And further we achieved the organizing pattern innovation and the operational optimization of our country large-scale sports event, under the backgrounds of our country's specific society, cultural and the sports event market environment.

At present, our country's large-scale sports event management level is not high so far, holding sports event is too depending upon the resources superiority which brings by whole nation system, the sports event benefit brought by management is still insufficient, the fundamental research is weak. In large-scale sports event management field, the fundamental research achievement relatively is still weak, did not pay enough attention to our country specific background system, lacks integration research and the empirical study. This obtained the expert and scholar's confirmation in the process of investigation.

Based on the research on materials combing with the inspection of the whole process of large-scale sports event organization and operational guidance, proposes the separation of "the organization 
pattern" and "the operational guidance pattern" of the large-scale sports event, includes "the sports event resources deployment" and "the sports event resources using" two sports event work category which contents and the nature are different.

Different with the overseas sports event goal pursuing sports event economic efficiency maximization, large-scale sports event held in our country mainly takes the social efficiency as the essential target, emphasized the large-scale sports event's public welfare attribute. Therefore, in our country large-scale sports event operational optimization aspect, we proposes sports event operational practice that can expand large-scale sports event social efficiency, like system construction large-scale sports event environmental management theory, large-scale sports event vivid landscape theory and so on. The thesis advanced the large-scale sports event operational process optimization theory--- Visualization. The Visualization theory is just in basic cognition of people in practical realm, and it is a universal suitable rule for improving managing level of mega multi-sports event.

\section{Technology Aggregation-diffusion Mechanism in Large-scale Sports Events}

The study of technology development mechanism in large-scale sports events is prevalent in the studies of technology industrialization and international development caused by the process of China's promoting High-tech Olympic strategy. For instance, Fang Fu-qian, as well as other scholars did the researches based on the life cycle theory, systematically analyzed the life cycle evolution of the technology cluster of the Olympic Games and its impact on the establishment of regional innovation system, and tried to explain the operation mechanism and fundamental rules of the development of technology industrial cluster in large-scale sports events. These research results provide more information for our further study of the operation mechanism of technology aggregation-diffusion model. Considering the process of technology aggregation and diffusion as a process of the flow of value, it is easy for us to draw a conclusion that it is a fundamental model of technology value aggregation-diffusion development with the conduction and increment of technology value as its driving forces. In other words, technology aggregation and diffusion is a special process of conducting and increasing the technology value, for it is built in the basic structure of the value chain.

At present, there are numerous research results of the value chain. The analysis and the study of the value chain has been the hot topic since first described. The traditional theories about the value chain mostly focused on the study of an enterprise's production process, even if on the international value chain which still pay much attention to a enterprise's economic production, but they still provide a new analysis paradigm for the study of technology value chain. When doing the researches on technology value chain, scholars start their study with the discussion of the technology innovation process, which is because that technology innovation is always developed in the field of corporations, but the more important is that it is the driving force of the development of modern corporations.

Some scholars do a special study on technology value chain and propose the concept of it. Science-Technology Value Chain is the aggregation of the innovation chain linked by a series of independent and related innovations which will increase the technology developing value in the whole process of developing from the technology innovation source to the industrialization of technology achievements. This concept is made from the industrial value creation point of view, but it is still suggestive owing to its analysis of the technology value chain as well as its creation process.

The implement of technology aggregation-diffusion development in large-scale sports events depends on the objectively existing intrinsic and extrinsic driving forces of conducting and adding the technology value. The intrinsic value is about technology's own, clue, which is created to complete some social work by the increasingly enrichment, advancement, and perfection of the technology system. While, the extrinsic value of technology is about the use value of the technology including its economic, social, and environmental values. Based on its own demands and the social demands, technology is applied to provide relevant products to cant' out the operation of the value chain, which will contribute to the realization of the two levels of conduction. The adding of technology's own value is based on technology system's characteristic of self-reinforcement. After reviewing the 
technology system in history and present, we find that the fundamental reason why technology is the great economic driving force of the society is that it is always able to build a relevantly dependent and efficient self-operating system according to its tasks and goals to be completed and reached.

\section{Some Suggestions on Organizing Large-scale Sport Event}

A sport event is a special event that has the characters of complicity, competition, multi-goals and objects and merchandise with strong organization culture and potential marketing background. A sport event can be classified into a project. It also supplies competition goods and relative service goods to meet various stakeholders' needs. Any sport event can be formed by three-dimensional description. Sport events have strong impact on society and culture, nature and environment, politic, tourism and economy on some circumstances. The process of sport event operation is to manage the input to output. Sport event operation must include the considerations of marketing that embraces goods marketing and service, humanization and environment, contingency and organizational behaviors. Operation tasks include budget and control, risk management, human resources management, logistics, communication, marketing and competition. Manageable factors are divided into two kinds, which include supporting factors and promoting factors.

The process of sport event operation is consisted of planning, organizing, implementing and evaluating. The model has two sub-models: one is a vertical model, which happens during the preparing time, the other is a horizontal model, which happens during staging time. Sport event's goal and objects decide the importance of each task. Integration marketing includes the sponsorship, advertisement, media broadcasting, courteous reception, public relationship and sales. Sport event operation model includes project start, planning and organizing, implementing and evaluating. Our country's sport event is affected by government's structure and mechanism, especially the competition institution, and manager's stuff. Although marketing operation methods must be researched fully. The Chinese operation model is received more attention, marketing in sport events includes project start planning and organizing, and implementing and evaluating.

\section{Conclusions}

Large-scale Sport Events organization and management is the key process for the work of Large-scale Sport Events, by using the method of project management can effectively manage, control and coordination of the organization and management of Large-scale Sport. Scientific and rational organization of large-scale competition is conducive to a successful match. It can enhance the image of the host and strengthen the competition between participants of the communication and learning from each other.

\section{References}

[1] Dianne Rahm. The role of information technology in building public administration theory, 1999.

[2] Federal Highway Administration, Managing Travel for Planned Special Events, Washington D.C., 2003.

[3] NCHRP. Transportation Planning and management for Special Events, A synthesis of highway practice. Transportation research Board. Washington D.C., 2003:32-41.

[4] ORTA. Nothing better than this (Transport for the Sydney 2000 Olympic and Paralympics Games). Sydney, Australia, OTRA, 2001.

[5] US Olympic Festival, After Action Report: Transportation Planning, Coordination and Operations, Oklahoma City, Ok. 1989. 